\title{
EMA Priority Medicines scheme (PRIME): will more paying-for-performance agreements be needed due to immature data?
}

\author{
F. Antoñanzas ${ }^{1} \cdot$ C. A. Juárez-Castelló ${ }^{1} \cdot$ R. Rodríguez-lbeas ${ }^{1}$
}

Published online: 29 November 2017

(c) Springer-Verlag GmbH Germany, part of Springer Nature 2017

The EU's centralized procedure for the authorization of a new drug is performed by the EMA. This well-known process requires the analysis of a large amount of data generated by clinical trials, as well as from other sources. This is a highly qualified activity and frequently becomes complex and needs additional input and requests from the pharmaceutical industry. In total, a maximum of 210 days can be devoted to the authorization of each product. Then, after this authorization, the dossier of the drug has to go on a pilgrimage to each Member State Ministry of Health's desk for price and reimbursement authorization. These bodies usually undertake additional assessments of the drug, establish its position in the domestic market, and finally authorize the product with either a negotiated price or a price that is set by the firm but always requires a public reimbursement decision. Some products are distributed through hospital pharmacies and then a further assessment is done by these centers in order to include them in their formularies. As HTA agencies are also common in many EU countries, their assessments may also be requested by some institutions before adopting decisions about the introduction of a new drug in the public service catalog. In total, since the application for the centralized authorization by EMA until the drug can be prescribed to a given patient, the process can easily take 24 months, and sometimes even longer.

All these activities can be understood as a reaction of public administrations to the monopolistic power that patent rights grant to manufacturers. In a pure unregulated monopolistic market, prices are fixed by companies and hence health authorities have to accept (as price takers) the imposed price. This is likely an undesired secondary effect of patent regulation, and it has no easy solution, as stated elsewhere [1,

F. Antoñanzas

fernando.antonanzas@unirioja.es

1 Department of Economics, University of La Rioja, 26004 Logroño, Spain
2]. Since last century, this reaction has taken the form of a control system that analyzes new chemical entities, checks benefits and risks for patients, and assesses the degree of innovation and improvement of the drug over the existing ones. This lengthy process has several drawbacks: it delays the beginning of sales for the firm (which means that the actual monopolistic power granted by the patents will be enjoyed for a shorter period) and some patients at health risk will not be treated as soon as they could otherwise be.

In order to overcome these drawbacks, health authorities have introduced different policies aiming to make market access more flexible, in fact bypassing the rather rigid administrative rules of the price and reimbursement process. These policies mostly apply to drugs addressing unmet crucial medical needs. In this sense, several decades ago, health care systems considered the introduction of still-nonauthorized-drugs through the so-called compassionate use that permitted prescription to selected patients under special conditions (mainly, for life-threatening diseases as soon as new drugs targeting them became available). Simultaneously, national health authorities also explored other mechanisms such as a kind of "fast track" assessment process to shorten the deadlines of the standard price and reimbursement decisions. The possibilities and combinations of legal procedures and policies addressing this problem across the EU countries during the last half century are far-reaching. In particular, the compassionate use approach has been widely applied by Member States (MS). However, the heterogeneity of formats of this policy makes the process cumbersome to pharmaceutical firms, meaning access is slower than it otherwise could be.

Late developments of effective and complex drugs in several therapeutic areas where life expectancy was really low have demanded a faster response by regulators so that patients could have a more homogeneous and rapid access to these drugs. In this sense, the European Medicine Agency (EMA) has implemented (March 2016) the PRIME (Priority 
Medicines) scheme combining three different approaches to deal with these issues: accelerated assessment, conditional marketing authorization, and compassionate use [3]. Briefly, as stated by EMA, accelerated assessment is "the rapid assessment of medicines in the centralized procedure that are of major interest for public health, especially ones that are therapeutic innovations. Accelerated assessment usually takes 150 evaluation days, rather than 210." On the other hand, conditional marketing authorization speeds up the access of drugs that address unmet medical needs. Mature comprehensive data is not required, prioritizing the health benefits of immediate access. Eligible drugs must either treat life-threatening diseases, be designated as orphan drugs, or intend to be used in emergency situations. Finally, compassionate use refers to the use, under strictly controlled conditions, of an unauthorized medicine in individual patients suffering from a life-threatening, long-lasting, or seriously debilitating disease for which a successful authorized treatment is not available. Each Member State coordinates and implements its own compassionate use program, setting the specific rules and procedures. The medicine may still be in the clinical trial phase, with no clear knowledge of its safety profile and dosage.

Notice that this set of policies seems to profile a win-win situation as patients and health care systems benefit faster from the innovations that effectively cure life-threatening conditions while at the same time giving pharmaceutical firms easier market access for their products. The results of the first year of this PRIME scheme were published recently. Between its launch in March 2016 and April 2017, EMA has considered 96 applications for PRIME. The majority of requests were submitted by small- and medium-sized enterprises. EMA had granted PRIME status to 21 medicines (22\%). Oncology and hematology, with six drugs each, were the most frequent therapeutic areas. The majority of the requests were in oncology (34 applications).

The conditional marketing authorization path was initiated in 2006, although it was later integrated within the PRIME scheme. By the year 2016, an evaluation of the 10 -year experience was published [4]. Remarkably, 35 drugs were granted that status and 13 of them were converted to the standard marketing authorization within that period; on average, it took 4 years for a drug to obtain such a conversion. Few therapeutic areas opted for CMA, oncology being the most common one. At the time of application, $53 \%$ of drugs only had response rate as the main endpoint of their efficacy and $34 \%$ were supported by data coming from single-arm studies. EMA imposed specific obligations upon the CMA grant, the most frequent request being the submission of clinical study results based on a longer duration and a larger sample. Most of the drugs observed the deadline submission of additional data and one-third of them even did it earlier (only $6 \%$ were delayed).
The applications for PRIME require that the drug not have entered the standard process for marketing authorization yet. As EMA states, firms may consider that their product will become eligible for this scheme during early stages of development (during the proof of concept or proof of principle) and then the dialog with the EMA officers will start. When data from clinical trials become available, the process would be simplified as the development process of the drug was already known-and perhaps accepted-by health authorities.

The UK started Brexit during spring 2016 and by October that year already had a parallel scheme named "Accelerated access" for its National Health System [5]. Importantly, although both initiatives mirror each other, the British one also gives the initiative to health authorities who in that case would indicate the medical needs of the system (i.e., life-threatening diseases without effective treatments) to the firms. If firms accept the suggestions and decide to innovate in those areas, then a fluent and permanent dialog with health authorities takes place, which guarantees that the product will have an accelerated market access despite the evidence not being totally mature by that time.

Interestingly, both the EMA's PRIME scheme and the UK's Accelerated access programs show that early dialog between health authorities and pharmaceutical companies is possible and plausible. Consequently, the industry can direct its research to areas of interest for the healthcare systems, thus gaining faster market access as a reward for its efforts. It would be interesting to also apply this new policy to drugs aimed at conditions that are more prevalent in lessdeveloped countries so that new treatments become available. The market reward of these innovations in terms of mark-ups is limited given the lower purchasing power of these countries; however, as the size of the global market is huge, the volume of expected sales would be large enough to provide an attractive benefit.

It is noteworthy that this more formal approach of PRIME to the problem of facilitating access to drugs for those patients under special needs merges with the rather less standard one of the risk-sharing agreements, which MSs already establish when needed. It is well known that MSs are responsible for price and reimbursement authorization and can, as a result, manage the prescriptions, recommending or limiting the utilization of drugs, and establishing special agreements with manufacturers, as public systems are the payers of drugs to a great extent (the difference between co-payment and price). These agreements usually adopt the common formats of paying-per-outcome, price-volume, or paying-per-efficiency and seek to reduce the uncertainties (mainly about the efficacy, safety, target population, and budgetary impact) that health authorities may face when assessing and managing a new drug. These contracts may allow firms to gain easier access to national markets. 
Generally speaking, drugs susceptible of risk-sharing agreements target patients with special needs whose health is seriously challenged by their diseases. In this sense, it can be anticipated that the higher the number of drugs with a granted status by PRIME, the larger the potential number of risk-sharing agreements at the MS level (at the regional or hospital level too, depending on how healthcare systems are organized), as there will be more drugs registered by EMA under the centralized procedure with immature information in the future. Both trends may offset each other (as the implementation of risk-sharing agreements also takes time) and the desired earlier or faster access will finally not be so successful. Some empirical data analysis is encouraged to check if these situations have happened in different EU markets since the PRIME scheme was initiated.

It is worth learning from the experience of the American Food and Drug Administration (FDA) as regards its accelerated process to approve drugs. Kim et al. [6] reviewed the approvals issued by FDA during the 5-year period (2008-2012) relating the path (accelerated vs. traditional) and the end points (surrogate-namely, progression-free survival and response rate vs. overall survival). All 15 drugs under the accelerated approval path used a surrogate point as the outcome measure while about half the drugs following the traditional path (21 out of 39) used that kind of measure. Authors concluded that only one drug of the accelerated path group was able to show a meaningful health outcome (overall survival) after 5 years of follow-up, while four drugs yielded that information in the traditional path. Kim et al. noted that the former were significantly more expensive. Furthermore, they stated that marketing authorization of most cancer drugs were based on surrogate endpoints (67\%, or 36 of 54). After several years of follow-up, they found that " $31(86 \%)$ of these approvals (57\% of the 54 drugs approved) had unknown effects on overall survival or failed to show gains in survival. In other words, most cancer drug approvals did not show to, or did not, improve clinically relevant end points".

This American analysis shows that post-marketing studies are needed to better understand the efficacy of approved drugs. This is especially applicable to early approved drugs such as those under the PRIME scheme, namely CMA and accelerated assessment, when mature data are still lacking.
Nevertheless, these drugs are highly priced within MSs, as they address unmet medical needs and have managed to generate a high expectation of their efficacy based only on surrogate endpoints. The experience of the EMA assessment of CMA only shows how companies perform in their communication with EMA and how many of the initially approved drugs are finally accepted under the standard marketing authorization. However, it is not clear whether some of these drugs have definitively provided patients with more solid outcomes (i.e., final end-points such as overall survival). In the absence of efficacy evidence after years of marketing authorizations, EMA and MS health authorities should be aware of this fact and review their initial decisions on positioning, pricing, and reimbursement—or, just in case, even remove that product from the market. As always, assessment is mostly needed to understand the real value of new drugs and, should it be uncertain, paying-for-performance policies would be recommended even for drugs granted that PRIME status.

Acknowledgements We are thankful for the financial support provided by the MINECO, project ECO2016-78685-R.

\section{References}

1. Antoñanzas, F., Juárez, C., Rodríguez, R.: Pharmaceutical patents, $\mathrm{R} \& \mathrm{D}$ incentives and access to new drugs: new ways of progress at the crossroad. Eur. J. Health Econ. 12(5), 393-395 (2011)

2. Antoñanzas, F., Rodríguez-Ibeas, R., Juárez, C.: Should the patent system for pharmaceuticals be replaced? A theoretical approach. Expert Rev. Pharmacoecon. Outcomes Res. 14(5), 617-626 (2014)

3. European Medicines Agency: PRIME: priority medicines. (2017). http://www.ema.europa.eu/ema/index.jsp?curl=pages/regulation/ general/general_content_000660.jsp. Accessed 9 Nov 2017

4. UK Government: Accelerated access review: final report. (2016). https://www.gov.uk/government/uploads/system/uploads/attachment_data/file/565072/AAR_final.pdf. Accessed 9 Nov 2017

5. European Medicines Agency: Conditional marketing authorisation: Report on ten years of experience at the EMA. (2017). www. ema.europa.eu/docs/en_GB/document_library/Report/2017/01/ WC500219991.pdf. Accessed 9 Nov 2017

6. Kim, C., Prasad, V.: Cancer drugs approved on the basis of a surrogate end point and subsequent overall survival: an analysis of 5 years of US Food and Drug Administration approvals. JAMA Intern. Med. 175(12), 1992-1994 (2015) 